\title{
Kernos
}

Revue internationale et pluridisciplinaire de religion grecque antique

19 | 2006

Varia

\section{Revue des Revues}

Vinciane Pirenne-Delforge et Angel Ruiz Pérez

URL : https://journals.openedition.org/kernos/512

DOI : $10.4000 /$ kernos.512

ISSN : 2034-7871

\section{Éditeur}

Centre international d'étude de la religion grecque antique

\section{Édition imprimée}

Date de publication : 1 janvier 2006

Pagination : 523-533

ISSN : 0776-3824

Référence électronique

Vinciane Pirenne-Delforge et Angel Ruiz Pérez, "Revue des Revues », Kernos [En ligne], 19 | 2006, mis en ligne le 22 mars 2011, consulté le 24 août 2022. URL : http://journals.openedition.org/kernos/512 ; DOI : https://doi.org/10.4000/kernos.512 


\title{
Revue des Revues
}

\author{
par Vinciane Pirenne-Delforge et Angel Ruiz PéREz
}

Cette chronique a été réalisée à Liège par Vinciane Pirenne-Delforge et à Santiago de Compostella par Angel Ruiz Pérez, pour les revues espagnoles. Toutes les références glanées au cours du dépouillement sont également mentionnées, sans que tous les articles en question aient pu être consultés, afin de fournir un parnorama le plus complet possible. Ce dépouillement concerne essentiellement les publications des années 2003 à 2005/6, auxquelles on a ajouté les tomes des revues portant un millésime antérieur mais seulement disponibles depuis peu. Nous remercions tous ceux qui pensent à nous envoyer leurs contributions sur la religion grecque.

Alganza Roldán Minerva, Maldonado F., « Las diosas del Reso », Florentia Iliberritana 16 (2005), p. 9-26.

ALLAN William, « Religious syncretism: the new gods of Greek tragedy », HSPh 102 (2004), p. 113155 [après une longue introduction sur la notion de syncrétisme, l'A. réserve davantage d'attention qu'à l'habitude aux « nouveaux dieux » sur la scène tragique, un thème qui nous informe sur les attitudes religieuses partagées par l'auditoire].

Alvar Jaime, Romero Recio Mirella, «La vie religieuse en mer », DHA supplément 1 (2005), p. 167-189 [l'exemple de la religiosité des marins montre que la « religion » des groupes sociaux ou professionnels n'est pas un ensemble imperméable, mais intégré à la cité].

ARTÉs Hernández José Antonio, « Magos en el Nuevo Testamento », MHNH 4 (2004), p. 5-31.

BARbantani Silvia, « Goddess of love and mistress of the sea. Notes on a Hellenistic hymn to Arsinoe-Aphrodite (P. Lit. Goodsp. 2, I-IV) », AncSoc 35 (2005), p. 135-165 [hymne copié au II s. de notre ère où le portrait d'Aphrodite correspond à la propagande royale ptolémaïque du $\mathrm{III}^{\mathrm{e}}$ s. av. J.-C.; diverses hypothèses, dont une sur le cadre d'exécution de l'hymne (un culte chypriote d'Arsinoé Philadelphe ?)].

BARRERA J.C.B., « Introduction to the logic of comparison in mythology », QS 62 (2005), p. 89-106.

BARRINGER Judith M., "The temple of Zeus at Olympia, heroes, and athletes », Hesperia 74 (2005), p. 211-241 [interprétation des frontons et des métopes du temple en fonction de la portée agonistique du sanctuaire : évocation de nikè plutôt que de dikè].

BASLEz Marie-Françoise, «Les Galles d'Anatolie: image et réalités », Res antiquae 1 (2004), p. 233-245.

Bautista, Hilario, «Antíoco de Atenas. Propuestas metodológicas para una edición crítica de su obra », MHNH 5 (2005), p. 157-176.

BEALl E.F., "Theism and mysticism in Hesiod's Works and Days », HR 43 (2004), p. 177-193 [cohérence et incohérence de la conception de Zeus et du divin dans l'œuvre].

Belayche Nicole, «Hypsistos. Une voie de l'exaltation des dieux dans le polythéisme grécoromain », ARG 7 (2005), p. 34-55 [analyse du dossier de l'épiclèse Hypsistos qui montre que l'exaltation de la divinité ainsi effectuée ne l'isole pas de ses collègues et ne fait pas nécessairement de l'appellation une variation païenne du monothéisme]. 
BENSEDDIK Nacéra, « Esculape et Hygie. Les cultes guérisseurs en Afrique », Pallas 68 (2005), p. 277-288 [le culte ne s'adressait pas particulièrement aux « masses »; réflexion sur les assimilations successives entre culte phénicien, grec et romain].

BetTini Maurizio, «Construire l'invisible. Un dossier sur le double dans la culture classique », Mètis N.S. 2 (2004), p. 217-230 [classification des doubles qui interviennent dans les mythes grecs, étude de leur composition et de leur consistance matérielle; comparaison avec les anges et démons qui ramène à l'eidôlon d'Hélène].

BETTINI Maurizio, «Mythos/Fabula: Authoritative and discredited speech », HR 45 (2005), p. 195 212.

BezzA G., «Per un lessico astrologico: glossario dei termini tecnici dell'Isagoge di Paolo d'Alessandria », MHNH 5 (2005), p. 277-305.

Biebuyck Benjamin, Praet Danny, Vanden Poel Isabelle, «The Eternal Dionysus. The Influence of Orphism, Pythagoreanism and the Dionysian Mysteries on Nietzsche's Philosophy of Eternal Recurrence ", Philologus 149 (2005), p. 52-77 [comment Nietzsche concevait-il ces courants et pourquoi a-t-il cessé d'y faire référence ensuite : cette question permet de monter comment il a évoqué, via la figure de Dionysos, des formes de transaction interculturelle d'où émerge une conception de l'existence humaine liée à la notion d'éternel retour].

Blanco Rodríguez Susana, « Ares. El dios más odiado del Olimpo griego », Minius 13 (2005), p. $15-26$

Bonnet Corinne, PIRENne-Delforge Vinciane, "'Cet obscur objet du désir' : la nudité féminine entre Orient et Grèce », MEFRA 116 (2004), p. 827-870 [comparaison des dossiers documentaires oriental et grec sur la nudité féminine; interrogation sur la portée de telles représentations en contexte religieux, notamment à Gortyne pour le volet grec].

Bravo García Antonio Pedro, «De nuevo sobre las uoces animalium y la magia greco-latina (esta vez con un pasaje cervantino al fondo) », MHNH 5 (2005), p. 127-156.

Breglia Luisa, «The Amphictyony of Calaureia », $A W 36$ (2005), p. 18-33 [examen des traditions mythiques liées à la participation d'Orchomène à la Ligue en regard des découvertes archéologiques récentes]

Bremmer Jan N., «A Macedonian Maenad in Posidippus (AB 44)», ZPE 155 (2006), p. 37-40 [l'épigramme funéraire de Niko évoque une jeune ménade macédonienne et témoigne d'une bonne connaissance du rituel ménadique de la part de l'auteur originaire de Pella].

Bricault Laurent, "Les dieux de l'Orient en Afrique romaine », Pallas 68 (2005), p. 289-309 [évaluation du succès de ces dieux, dont Cybèle, Mithra et les divinités du cercle isiaque].

Brigger Eliane, Giovannini Adalberto, « Prothésis : étude sur les rites funéraires chez les Grecs et chez les Étrusques », MEFRA 116 (2004), p. 179-248 [analyse exhaustive et approfondie de l'ensemble de la documentation littéraire et iconographique sur la prothésis, interprétée comme l'exposition du défunt sous une tente dressée pour l'occasion; comparaison avec la structure des tombes de Tarquinia].

Burgess Jonathan S., «Untrustworthy Apollo and the destiny of Achilles: Iliad 24.55-63 », HSPh 102 (2004), p. 21-40 [une interprétation du passage homérique y voit une prophétie funeste qu'Apollon aurait chantée aux noces de Thétis et Pélée, parallèlement au fr. 350 Radt d'Eschyle; l'A. dissocie les deux textes et contexte que le premier serait une allusion à une tradition dont Eschyle serait un représentant ultérieur].

Burns Dylan, «Proclus and the theurgic liturgy of pseudo-Dionysius », Dionysius 22 (2004), p. 111-132.

Burrell Barbara, «Iphigeneia in Philadelphia », Classical Antiquity 24 (2005), p. 223-256 [une monnaie de Philadelphie en Lydie offre l'image d'Iphigénie, Oreste et Pylade installant l'Artémis taurique, identifiée à l'Artémis Anaitis locale; ses liens avec l'Artémis d'Éphèse ont permis à la cité de revendiquer une place équivalente à celle de ses puissantes voisines].

ByL Simon, «L'Hymne homérique à Déméter, les Nuées et Éleusis », BAGB (2006), p. 84-99 [mise en parallèle systématique des deux ouvres afin de mettre au jour des 《 points de contact »]. 
CAIRns Douglas, " Myth and the polis in Bacchylides' eleventh Ode », JHS 125 (2005), p. 35-50 [la dimension mythique de l'ode est exploitée par l'A. en vue d'inscrire la victoire d'Alexidamos de Métaponte dans le contexte de l'éloge de sa cité en tant que fondation coloniale, divinement favorisée, notamment par Artémis].

CALDERÓn DORDa Esteban, « Rito y sacrificio en Esquilo. Aspectos léxicos », Itaca 19 (2003), p. 925.

Calvo MarTínez José Luis « El Himno xaī@ Ecáxu», a Helios, del papiro parisino. Edición crítica con introducción y comentario », MHNH 4 (2004), p. 265-278.

CALVO MARTínez José Luis, « ¿Licnomancia o petición de demon páredros? edición con comentario de fragmentos hímnicos del PGM I 262-347 », MHNH 5 (2005), p. 263-275.

CAmpos Daroca Javier, « Una nota mitológica al texto de Máximo de Tiro », CFC (G) 15 (2005), p. $151-154$

CARBON Jan-Mathieu, « $\Delta \alpha \dot{\alpha} C \omega \omega v$ and $\delta \alpha i ́ \omega v:$ a new inscription from Mylasa », EA 38 (2005), p. 1-6 [dédicace d'un autel au daimôn de Demorkitè par une phratrie de Mylasa, qui était sans doute une association cultuelle plutôt qu'une subdivision civique et portait le nom d'un daimon macédonien, Darrôn; l'inscription mêle les éléments religieux cariens et non cariens].

CATONi Maria Luisa, «Le regole del vivere, le regole del morire. Su alcune stele attiche per donne morte in parto », $R A$ (2005), p. 27-53 [lecture du schéma iconographique utilisé et typologie des scènes; comparaison avec la peinture à sujet mythologique, comme «l'accouchement » de Sémélè].

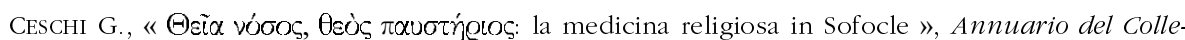
gio Arcivescovile 'C. Endrici' di Trento, 70 (2003-2004), p. 51-59.

CHANiOTIS Angelos, «Under the watchful eyes of the gods: divine justice in Hellenistic and Roman Asia Minor », YCS 31 (2002), p. 1-43

CHANKowski Véronique, «Techniques financières, influences, performances dans les activités bancaires des sanctuaires grecs », Topoi 12/13 (2005), p. 69-93.

Chasson Charles C., « Myth, ritual, and authorial control in Herodotus' story of Cleobis and Biton (Hist. 1.31) », AJPh 126 (2005), p. 41-64 [Hérodote aurait introduit sciemment des éléments de mythe et de rituel initiatiques dans le récit; la dédicace de statues à Delphes renvoie à la fois à l'accès à la classe des adultes et à la préservation du kleos].

CHESHIRE Keyne, « Progression and Unity in Callimachus' Hymn to Apollo », CJ 100 (2005), p. 331348.

ClARKE Michael, «An ox-fronted river-god. Sophocles, Trachiniae 12-13 », HSPh 102 (2004), p. 96-112 [ces deux vers ouvrent la voie à une réflexion sur la combinaison des parts humaine et animale, et son évolution dans la tradition littéraire et iconographique].

CUllyer Helen, " A wind that blows from Thrace: Dionysus in the fifth stasimon of Sophocles" Antigone », CW 99 (2006), p. 3-20 [les allusions géographiques à la Thrace suggèrent que la présence du dieu à Thèbes est aussi invisible et destructive que le vent de l'eros].

D'Acunto M., «Il tempio di Apollo a Dreros: il culto e la 'cucina del sacrificio' », $\operatorname{AION(ArCh)}$ N.S. 9-10 (2002-2003), p. 9-62

DAumas Michèle, «De Thèbes à Lemnos et Samothrace. Remarques nouvelles sur le culte des Cabires », Topoi 12/13 (2005), p. 851-881 [réaction de l'A. aux critiques dirigées contre son ouvrage Cabiriaca et aux publications récentes sur les cultes à mystères].

DAvies Malcolm, « Dolon and Rhesus », Prometheus 31 (2005), p. 29-34.

DAvies Malcolm, « The Sirens at mid-day », Prometheus 31 (2005), p. 225-228.

DEBORD Pierre, «La déesse Ma et les hirondelles blanches », REG 118 (2005), p. 15-30 [étude d'une plaque sur un tombeau de Cappadoce menaçant les contrevenants de représailles].

Deschamps Lucienne, "Quelques hypothèses sur le 'calendrier de Thysdrus' », REA 107 (2005), p. 103-130 [l'examen de la mosaïque dite «calendrier de Thysdrus » (début III s. ap. J.-C.) suggère que le propriétaire de la maison où on l'a découverte était un païen cultivé et mystique, peut-être initié aux mystères de Dionysos et d'Osiris] 
DESPINIS Giorgos I., «Die Kultstatuen der Artemis in Brauron », MDAI(A) 119 (2004), p. 261-315 [mise en perspective des inventaires de l'acropole ( 5 exemplaires de statue cultuelle) et des restes statuaires mis au jour à Brauron (4 acrolithes très fragmentaires)].

DESPINIS Giorgos, « Der Dionysos-Altar in Brauron », JDAI 119 (2004), p. 41-65 [l'autel rond à relief en marbre de Brauron est ici daté autour de 400 av. J.-C. et interprété comme la représentation de la réception de Dionysos en tant que « dieu visiteur » au sanctuaire d'Artémis].

DIEZ DEL CORRAL, Pilar, «El rapto: ¿una forma de amor? una interpretación de las imágenes de persecución y rapto de Dionisio y Ariadna », Gallaecia 24 (2005), p. 75-97.

Díez Platas, Fátima, «Sobre ninfas y heroínas en la épica griega arcaica », Fortunatae 14 (2003), p. 11-26.

DONDERER Michael, « Das Heraion in Olympia und sein Säulenkranz », BABesch 80 (2005), p. 7 20.

DREW GRIFFITH R., « Gods' blue hair in Homer and in eighteenth-dynasty Egypt », CQ 55 (2005), p. 329-334 [poussant à son terme une suggestion de Ch. Picard, l'A. démontre que l'usage de xúavoc pour qualifier les cheveux, les sourcils et la barbe des dieux serait inspiré par les productions de l'art égyptien et l'usage du lapis-lazuli à cette fin].

DrozDEK Adam, « Anaximenes: Theology and Physics », Eranos 102 (2004), p. 40-45.

DROZDEK Adam, «Euclides of Megara: god = phronesis = good », AAntHung 45 (2005), p. 27-34.

DROZDEK Adam, « Protagoras and Instrumentality of Religion », AC 74 (2005), p. 41-50 [Protagoras comprend la religion en tant que pratique sociale et comme outil dans les mains des dirigeants].

DrozDEK, Adam, « Sceptics and a religious instinct », Minerva 18 (2005), p. 93-108.

DuHoux Yves, «Les nouvelles tablettes en linéaire B de Thèbes et la religion grecque », AC 74 (2005), p. 1-19 [contre les interprétations des tablettes par leurs éditeurs, l'analyse linguistique constate que 1) il n'existe aucun élément textuel étayant l'existence d'une triade et a fortiori éleusinienne, 2) les trois membres de la prétendue triade sont probablement des humains, 3) les « animaux sacrés » n’ont pas la moindre existence démontrable].

EHLING Kay, « Ein reitender Telesphoros », EA 35 (2005), p. 159-164 [analyse d'un relief en mauvais état, provenant d'Asie Mineure et représentant un Telesphoros cavalier].

EHRHARDT Wolfgang, «Zu Darstellung und Deutung des Gestirngötterpaares am Parthenon », JDAI 119 (2004), p. 1-39 [la représentation partielle du couple Hélios et Séléné, dans la décoration du Parthénon et sur les bases de l'Athéna et du Zeus de Phidias fait de leur cycle sans fin un trait essentiel de l'ordre olympien].

EKROTH Gunnel, «Blood on the altars? On the treatment of blood at Greek sacrifices and the iconographical evidence ", $A K 48$ (2005), p. 9-29 [l'iconographie des vases n'offre guère l'image d'autels couverts de sang et les exceptions s'expliquent du fait que le sang doit être jeté ou par la portée mythologique des scènes; ce constat renforce l'hypothèse - fondée sur les inscriptions et la littérature - de l'utilisation du sang dans la cuisine sacrificielle].

Esteban SAnTos, Alicia, « Mujeres terribles (Heroinas de la mitología griega) I », CFC (G) 15 (2005), p. 63-93

Evans N.A., « Feasts, citizens, and cultic democracy in classical Athens », AncSoc 34 (2004), p. 125 [mise en perspective des interprétations divergentes sur la question de la participation des femmes à la thusia].

FARAONE Christopher A. "In the Horn of an Ox. A Curious Hexametrical Curse from Hellenistic Cyrene (SGD 150) », MHNH 4 (2004), p. 51-62

FORSDYKE Sara, "Revelry and riot in Archaic Megara: Democratic disorder or ritual reversal? », JHS 125 (2005), p. 73-92 [la prétendue existence d'une démocratie radicale à Mégare au milieu du $\mathrm{VI}^{\mathrm{e}}$ s. av. J.-C. serait le résultat d'une mauvaise interprétation par les modernes de rites traditionnels d'inversion et de transgression].

GÄRTNER Thomas, «Der mythische Held in saekularisierter Umgebung: Zum Orestes des Euripides », Prometheus 31 (2005), p. 1-28. 
GASPARRO Domingo, MORET Jean-Marc, «Dessins sans dessein : une clé de lecture simple pour les métopes 13-20 Sud du Parthénon », RA (2005), p. 3-25 [nouvelle lecture des métopes : 13-14, les Cécropides; 15-16, Érechthée et son adversaire; 19-20, les Pandionides; 17 : Borée enlevant Orithyie; histoire d'Athènes et rythme calendaire des grandes fêtes].

GHEZZI Vania, «I tiranni siracusani e le vergine locrese », PP 338 (2005), p. 321-360 [reprise du dossier de la prétendue " prostitution rituelle » locrienne].

GIORDANO-ZECHARYA Manuela, «As Socrates shows, the Athenians did not believe in gods », Numen 52 (2005), p. 325-355 [réévaluation de la notion de « croyance » en contexte athénien sur la base du verdict posé contre Socrate qui montre que la dimension rituelle de la religion de la polis est centrale dans l'accusation contre le philosophe; analyse de l'historiographie du procès de Socrate et des raisons qui ont conduit à privilégier la notion de « croyance »].

GIORDANO-ZECHARYA Manuela, «Ritual appropriateness in Seven against Thebes. Civic religion in a time of war ", Mnemosyne 49 (2006), p. 53-74 [analyse de la tension entre deux attitudes religieuses opposées, selon qu'elles sont assumées par les hommes ou les femmes; la prière des premiers est rituellement pertinente et la lamentation des secondes ne l'est pas].

GogGaKI Konstantina, "The athletic victory as a value in the Pindaric ode », Nikephoros 17 (2004), p. 123-134 [la valeur dont il est ici question relève à la fois d'une dimension intellectuelle, spirituelle, religieuse et politique].

GONZALES Matthew, "The oracle and cult of Ares in Asia Minor », GRBS 45 (2005), p. 261-283 [confrontation entre la référence d'Hérodote (VII, 76-77) à cet oracle et un petit corpus épigraphique qui atteste la portée oraculaire du dieu et la popularité de son culte dans le sud de l'Asie Mineure.

GonzÁlez GonzÁlez Marta, Macías Gil Cristóbal, «El Banquete de Metodio de Olimpo y sus argumentos contra la astrología », MHNH 5 (2005), p. 307-341.

GORRINI M.E., «Eroi salutari della Grecia continentale tra istanze politiche ed universali », AION(Arch) N.S. 9-10 (2002-2003), p. 163-191.

GREENE Elizabeth S., "Revising illegitimacy: the use of epithets in the Homeric Hymn to Hermes ", CQ 55 (2005), p. 343-349 [l'usage des épithètes permet au poète de créer, de prédire et de confirmer le rôle de la divinité et de définir sa position parmi les Olympiens].

GrotTANelli Cristiano, «Fruitful Death: Mircea Eliade and Ernst Jünger on human sacrifice, 1937 1945 », Numen 52 (2005), p. 116-145 [comparaison de l'idéologie sacrificielle de ces deux intellectuels et réflexion sur l'entrecroisement complexe entre pouvoir politique, conflit violent, dans un âge marqué par le fachisme, et les thèmes religieux traditionnels comme l'antique pratique sacrificielle des Grecs]

GUIMIER-SORBETS Anne-Marie, « Dionysos dans l'andrôn. L'iconographie des mosaïques de la maison grecque au $\mathrm{IV}^{e}$ et au $\mathrm{III}^{\mathrm{e}}$ siècle avant J.-C. », MEFRA 116 (2004), p. 895-932 [corpus de 103 pavements dont le décor floral suggère métaphoriquement la présence du dieu, à mettre en relation avec la libation et/ou la présence du cratère au milieu des convives].

Hamon Patrice, «Rites et sacrifices célébrés dans le conseil. Remarques sur les cultes du bouleutèrion et leur évolution à l'époque hellénistique », Topoi 12/13 (2005), p. 315-332.

HARDIE Alex, «Sappho, the Muses, and life after death », ZPE 154 (2005), p. 13-32 [interprétation du «nouveau papyrus » de Sappho, qui permet d'enrichir l'interprétation de sa poésie touchant à la question de la mortalité et des bénéfices eschatologiques escomptés de la participation chorégique à son « association cultuelle » sous l'égide des Muses].

HEATH John, « Blood for the dead: Homeric ghosts speak up », Hermes 133 (2005), p. 389-400 [examen du rôle de l'absorption de sang par les morts dans l'épopée, essentiellement du point de vue de la fonction littéraire du motif].

HillgRuber Michael, « Medea, Jason und Thetis. Zur Gründungslegende des Aphroditetempels von Korinth », MH 62 (2005), p. 65-69 [reconstitution de deux versions du mythe de fondation, fondée sur des allusions des allusions liées à la transmission de l'épigramme de Simonide sur la supplication des femmes de Corinthe à Aphrodite en 480]. 


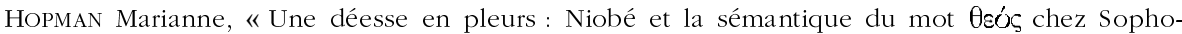
cle », REG 117 (2004), p. 447-467 [le statut de « déesse » attribué à Niobé (Ant., 834; Él., 150) est problématique et interprété ici comme une référence au statut cultuel « héroïque » du personnage, ainsi que l'attestent d'autres emplois chez Sophocle].

DE Hoz María Paz, « Literacy in rural Anatolia: the testimony of the confession inscriptions », $Z P E$ 155 (2005), p. 139-144 [même si l'approche de ces textes n'est pas ici religieuse, l'analyse littéraire et linguistique a des implications religieuses, notamment en terme de vecteurs de « propagande religieuse »].

Huet Valérie, Lissarague François, «Un relief 'néo-attique' : Icarios, le retour », Mètis N.S. 3 (2005), p. 85-100 [mise en contexte - tant historiographique qu'historique - du motif de la visite de Dionysos chez un banqueteur appartenant à cette catégorie stylistique problématique qu'est le « néo-attique », qui correspond à un usage bien réel des Romains].

Hummel Pascale, «Langue(s) rituelle(s), formulaire collectif et formulaire individuel dans la littérature grecque archaïque ", Maia 57 (2005), p. 265-275 [réflexion sur l'appropriation poétique du patrimoine mythologique en fonction avec la grille de lecture du "formulaire », conçu comme l'empreinte laissée par le mythe dans la parole poétique].

JAILlARD Dominique, «'Images' des dieux et pratiques rituelles dans les maisons grecques. L'exemple de Zeus Ktésios », MEFRA 116 (2004), p. 871-893 [étude des rites qui " présentifient » le dieu dans l'espace domestique, assortie d'une réflexion sur les formes et fonctions des vases du type kados et sur la notion de ktésis dans l'économie domestique].

JAILlarD Dominique, «Mises en place du panthéon dans les Hymnes homériques. L'exemple de l'Hymne à Déméter », Gaia 8 (2005), p. 49-62 [le poème donne à voir le panthéon du point de vue de la déesse et, par les fictions qu'il suscite, explore les fonctions qu'elle y assume].

JANAKIEva Svetlana, « Noces prolongées dans l'Hadès: d'Évadné aux veuves thraces », RHR 222 (2005), p. 5-23 [comparaison du thème de la mort comme mariage, dans des mythes grecs, des coutumes thraces et d'autres peuples i.-e.; à la source de cette conception se trouve la croyance en une vie dans l'au-delà et en la continuité du mariage dans l'Hadès].

JOURDAIN-ANNEQuin Colette, «Les divinités orientales dans les Alpes », DHA supplément 1 (2005), p. 191-189 [étude du culte de Mithra et mise en perspective de l'attrait exercé par les grandes divinités féminines comme Isis et Cybèle, fonctionnellement proches des " Mères » gauloises].

JOURDAN Fabienne, « Manger Dionysos. L'interprétation du mythe du démembrement par Plutarque a-t-elle été lue par les néo-Platoniciens ? », Pallas 67 (2005), p. 153-174 [Plutarque est le premier témoin de l'épisode mythique de la dévoration de Dionysos par les Titans; l'exégèse philosophique qu'il en donne est tout à fait originale en contexte néo-platonicien; il l'a peutêtre « inventé », càd « découvert » pour les besoins de son plaidoyer pro-végétarien].

KAIZER Ted, «In Search of Oriental Cults. Methodological problems concerning 'the particular' and the 'general' in Near Eastern religion in the Hellenistic and Roman periods », Historia 55 (2006), p. 26-47 [réflexions de méthode qui sont transposables avec profit aux problématiques du 'local' et du 'panhellénique' en contexte grec].

KAJAVA Mika, « Hestia: Hearth, Goddess, and Cult », HSPh 102 (2004), p. 1-20 [constat de l'absence de prêtrise explicitement associée à Hestia et discussion d'un petit corpus d'inscriptions

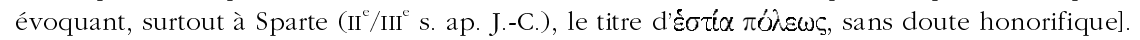

KeEsLING Catherine M., « Misunderstood gestures: Iconatrophy and the reception of Greek sculpture in the Roman imperial period », Classical Antiquity 24 (2005), p. 41-79 [les dédicaces de statues archaïques et classiques mentionnent le dédicant, la divinité et le sculpteur, sans identifier le sujet représenté ou l'occasion de la dédicace; l'exemple de la statue de Milon de Crotone à Olympie, de la Léaina et de l'Athéna Hygieia de l'acropole d'Athènes montre comment ces « trous » ont été comblés par une tradition orale].

KEESLING Catherine M., «Patrons of Athenian votive monuments of the Archaic and Classical Periods », Hesperia 74 (2005), p. 395-426 [étude du rôle joué par des patrons privés dans la production d'art et d'inscriptions à Athènes en ces périodes, dans le cadre de la religion votive : analyse d'offrandes à dédicataires multiples]. 
KORENJAK Martin, «'Unbelievable Confusion'. Weshalb sind di 'Hieroi Logoi' des Aelius Aristides so wirr? », Hermes 133 (2005), p. 215-234 [outre l'arrogance rhétorique du temps, l'analyse met en relief le parallèle sciemment orchestré entre les énoncés d'Asclépios et ceux d'Aelius Aristide].

KossaïrI Christine, «Daphnis et Daphné. Mythe et poésie dans l'Idylle I de Théocrite », BAGB (2005), p. 113-144

KOUSSER Rachel, «Creating the past: the Vénus de Milo and the Hellenistic reception of Classical Greece », AJA 109 (2005), p. 227-250 [mise en évidence du contexte concret (un gymnase) et mythologique (le Jugement de Pâris) dans lequel s'inscrivait cette statue; l'analyse enrichit notre connaissance la réception hellénistique de la sculpture et des mythes classiques].

KuRKE Leslie, "Choral lyric as 'ritualization': Poetic sacrifice and poetic ego in Pindar's sixth Paian », Classical Antiquity 24 (2005), p. 81-130 [l'ego exceptionnel du poète dans cette pièce proviendrait de la présence de deux chœurs, l'un delphique, l'autre éginète, le poète devenant dès lors la figure médiatrice entre deux communautés et deux traditions mythiques].

LAMBERT Stephen D., "Athenian state laws and decrees, 352/1 - 322/1: II. Religious regulations », ZPE 154 (2005), p. 125-159 [prolégomènes au fasc. 2 de $I G \mathrm{II}^{3}$ qui reprend les lois et décrets de la cité concernant les affaires religieuses - donc ni les décrets honorifiques pour desservants ni les textes religieux comme les « calendriers »].

LARSON Jennifer, « Lugalbanda and Hermes », CPh 100 (2005), p. 1-16 [étude des correspondances entre l'épopée néo-sumérienne de Lugalbanda et les 150 premiers vers de l'Hymne ps. hom. à Hermès, pour conclure à l'influence de la première sur le second].

LAURIOLA Rosanna, «Athena and the Paphlagonian in Aristophanes' Knights. Re-considering Equites 1090-5, 1172-81 », Mnemosyne 49 (2006), p. 75-94 [examen du traitement d'Athéna dans ces deux passages, notamment les épithètes utilisées].

LE Roy Christian, « Lieux de mémoire en Lycie », Cabiers Glotz 15 (2004), p. 7-15 [la civilisation lycienne a sciemment élaboré, entre les $\mathrm{V}^{e}$ et $\mathrm{II}^{\mathrm{e}} \mathrm{s}$. av. J.-C., une tradition mythique et une organisation de l'espace cultuel permettant de conserver une identité anatolienne].

LEBessi Angeliki, MuHly Polly, PAPASAVvas George, «The Runner's Ring, a Minoan Athlete's Dedication at the Syme Sanctuary, Crete », MDAI(A) 119 (2004), p. 1-31.

LIVREA E., « Lycidas and Apollo in Theocritus' 'Talysia' », Eikasmos 15 (2004), p. 161-167.

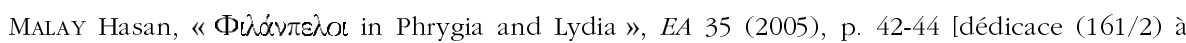

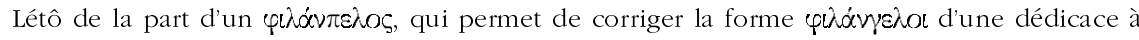
Hosios et Dikaios, et donc de supprimer l'entrée du LSJ rev. suppl.].

MARTín HERNÁNDEZ Raquel, « La muerte como experiencia mistérica. Estudio sobre la posibilidad de una experiencia de muerte ficticia en las iniciaciones griegas », Ilu 10 (2005), p. 85-105.

MATSAS Dimitris, Dimitrova Nora, « New Samothracian inscriptions found outside the sanctuary of the Great Gods », ZPE 155 (2005), p. 127-136 [notamment des listes d'initiés].

MaUcourant Jérôme, « À propos de l'économie des sanctuaires dans l'Antiquité : une perspective institutionnaliste », Topoi 12/13 (2005), p. 117-132.

Mecu M., « Lucien athée. Réflexions autour d'un topos », Studii clasice 37-39 (2001-2003), p. 103130.

MEDDA Enrico, « Aristofane e un inno a rovescio: la potenza di Pluto in Pl. 124-221 », Philologus 149 (2005), p. 12-27 [la prière parodique de ce passage renverse la relation entre homme et dieu qu'impliquent normalement les hymnes et les prières].

MONTEPAONE Claudia, «Où l'on revient sur la monnaie de fer spartiate », Mètis N.S. 2 (2004), p. 103-123 [retour sur la faucille sacrificielle comme signe prémonétaire dans le cadre du culte d'Artémis Orthia et de l'Artémis Taurique].

MOUSTAKA Aliki, « Neue lakonische Kratere aus dem samischen Heraion », MDAI(A) 119 (2004), p. 55-66 [mise en perspective du motif guerrier du cratère avec d'autres ex-voto du sanctuaire, comme des armes ou des boucliers votifs]. 
MUÑIZ GRIJALVO Elena, "Elites and religious change in Roman Athens », Numen 52 (2005), p. 255-282 [étude de l'accroissement du pouvoir religieux des élites, du succès de certains dieux comme Asclépios, Isis, Zeus Hypsistos, et de la relation entre les deux phénomènes].

NicOLAI Walter, «Sind personale und apersonale Gottesvorstellung miteinander vereinbar oder nicht? », GB 24 (2005), p. 15-29.

OAKLEY John H., ReITZAMMER Laurialan, «A Hellenistic terracotta and the gardens of Adonis ", JHS 125 (2005), p. 142-144 [une «tanagra » mise au jour à Myrina en Éolide est interprétée comme une participante aux Adonies versant une libation parfumée dans un « jardin » d'Adonis].

OBbink Dirk, «A new Archilochus poem », ZPE 156 (2006), p. 1-9 [exploitation du mythe de Télèphe].

OIKONOMOPOULOU, Polytimi, «Pharmaka and Witches », MHNH 4 (2004), p. 123-139.

OSANNA Massimo, "Rituali sacrificali e offerte votive nel santuario lucano di Torre di Satriano ", $A R G 6$ (2004), p. 45-62 [l'analyse des données de la fouille aboutit à une mise en perspective du rituel sacrificiel dont la méthode et les résultats sont exploitables en contexte grec].

PAKKANEN Jari, «The Erechtheion construction work inventory ( $\left.I G 1^{3} 474\right)$ and the Dörpfeld temple », AJA 110 (2006), p. 275-281 [contestation de l'article de G. Ferrari, AJA 106 (2002), p. 11-35, qui faisait l'hypothèse du maintien en l'état, sur l'acropole athénienne, d'une partie du sanctuaire d'Athéna endommagé par les Perses, jusqu'en pleine période romaine].

PaluChOWsKi Adam, « Note au sujet des Velchania de Lyttos (ICret, I, 18 Lyttos, n 11) », DHA 3331 (2005), p. 25-32 [l'article soutient la succession Theodaisia et Velchania dans le calendrier festif crétois, contrairement à l'ordre inverse préconisé par G. Capdeville, Volcanus (1995); ce changement a des implications pour l'interprétation de la fête].

PÀmIAS Jordi, « Dionysus and donkeys on the streets of Alexandria: Eratosthenes' criticism of Ptolemaic ideology », HSPh 102 (2004), p. 191-198 [mise en perspective des attaques larvées d'Ératosthène contre le cœur "dionysique » de l'idéologie religieuse alexandrine en deux passages de ses Catastérismes (Cat., 11 \& Fragm. Vat., 24)].

Perea YéBenes Sabino, «Un capítulo de la teúrgia antigua: los oráculos de Hécate y la cuestión de las estatuas parlantes », MHNH 5 (2005), p. 189-240.

PÉREZ JIMÉNEZ Aurelio «Influencias astrales en la fundación de ciudades y en las tareas de construcción », MHNH 4 (2004), p. 173-196.

PerotTi, Pier Angelo, « Polifemo in Omero, Euripide, Luciano », Minerva 18 (2005), p. 39-70.

PETZL Georg, « Furchterregende Götter? Eine Notiz zu Diogenes von Oinoanda NF 126 », ZPE 153 (2005), p. 103-107.

PICARD Olivier, «Les $\chi \bigcup ̛ ́ n u x \tau \alpha$ d'Apollon et les débuts de la monnaie à Delphes », Topoi 12/13 (2005), p. 55-68.

PIRENNE-Delforge Vinciane, «Image des dieux et rituel dans le discours de Pausanias : de 1'axiologie' à la 'théologie' ", MEFRA 116 (2004), p. 811-825 [au départ d'une brève étude lexicale, analyse du lien que Pausanias opère entre la statue comme objet « digne d'être vu » et la dimension religieuse que la plupart des statues avaient conservée lors de sa visite].

Pombo GAllego Nuria, « Historiografía de la religión minoica », Gallaecia 25 (2006), p. 347-358.

PONT Anne-Valérie, « Le paysage religieux grec traditionnel dans les cités d'Asie Mineure occidentale, au $\mathrm{IV}^{\mathrm{e}}$ et au début du $\mathrm{V}^{\mathrm{e}}$ siècle », REG 117 (2004), p. 546-577 [panorama de la survie d'espaces sacrés au coeur de l'espace urbain à une date avancée et recensement de toute une série de marques discrètes de l'ancienne religion].

PRÊTEUX Franck, «Priapos Bébrykès dans la Propontide et les Détroits : succès d'un mythe local », REG 118 (2005), p. 246-265 [synthèse sur l'origine du culte de Priape, en liaison avec la mémoire du peuple disparu des Bébryces; réflexions sur sa fonction de protecteur des marins et sur le parallèle des cultes de Zeus Ourios et de Stomianos dans cette fonction].

Pucci Pietro, "Dynamics and variations of Nietzsche's couple », Mètis N.S. 2 (2004), p. 163-185 [analyse de l'invention métaphorique de Nietzsche dans la Naissance de la Tragédie et de la 
critique récente de la tragédie qui se réfère encore au thème de l'opposition entre Apollon et Dionysos (Dodds, C. Diano, N. Loraux)].

RASMUSSEN Tom, «Herakles' apotheosis in Etruria and Greece », $A K 48$ (2005), p. $30-39$ [1'adoption d'Héraclès adulte par Héra qui l'allaite, telle que la figurent 4 miroirs étrusques n'est pas nécessairement un épisode étrusco-italique du mythe : cette adoption rituelle semble connue dans le répertoire mythologique grec, mais sans être représentée dans l'art grec lui-même].

REED Jay, "The fruits of Adonis », Philologus 149 (2005), p. 362-364 [discussion d'une glose de la

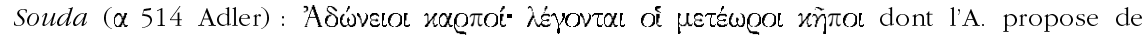
corriger $x \hat{n} \pi 0 t$ en $x \alpha \varrho \pi 0 i ́]$.

RIVES J.B., « Phrygian tales », GRBS 45 (2005), p. 223-244 [la référence aux Phyrgiens, en tant qu'antique peuple dont le langage était originel, permettait de faire remonter haut dans le temps les procédés d'interprétation des mythes comme l'allégorie et l'évhémérisme].

Rocchi Maria, «Culti sui monti della Grecia. Osservazioni da una lettura di Pausania », $A R G 7$ (2005), p. 56-61 [relation entre montagnes et cultes en Grèce, et tout particulièrement dans les descriptions de Pausanias].

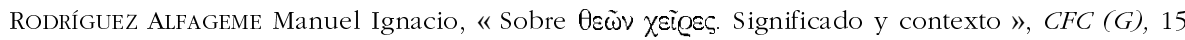
(2005) p. 125-140.

RODRÍGUEz CidRE Elsa, «Animalizar a la víctima: Políxena en la Hécuba de Eurípides », Veleia 21 (2004), p. 99-108.

ROMERO Becerra Daniel, "Creencias y utilidades sobre las setas y los hongos en el mundo antiguo: entre lo real y lo imaginario », Gallaecia 25 (2006), p. 333-346.

RUIZ DE ElVIRA PRIETO Antonio, « La muerte de Eurídice: sobre el hidro, el quersidro y el quelidro », CFC (L) 24 (2004), p. 239-247.

Ruscu Ligia, «Two Pontic notes », EA 35 (2005), p. 125-130 [dédicace d'un autel au Theos Hypsistos par une certaine Valéria; cela fait monter à quatre les occurrences de ce dieu dans le corpus des inscriptions de Sinope].

ŞAHIN M. Çetin, « A Hellenistic list of donors from Stratonikeia », EA 35 (2005), p. 9-11 [inscription du début du II ${ }^{\text {e }}$ s. av. J.-C., qui mentionne des donations peut-être effectuées pour des mystères de Déméter].

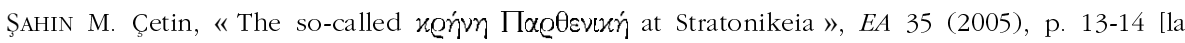
fontaine porte ce nom car une statue d'Athéna Parthenos monumentale s'élevait à côté].

SCHEID John, «Un élément original de l'identité romaine : les cultes selon le rite grec », Mètis N.S. 3 (2005), p. 25-34.

SCHMIDT-DounAs Barbara, "Frühe Peripteraltempel in Nordgriechenland », MDAI(A) 119 (2004), p. 107-145 [ce type architectural, attesté pour le temple de Thermi, de la Parthenos à Neapolis près de Kavalla et d'Héraclès à Thasos est directement inspiré de l'Asie mineure].

SEBillotTe Cuchet Violaine, «La sexualité et le genre: une histoire problématique pour les hellénistes. Détour par la 'virginité' des filles sacrifiées pour la patrie », Mètis N.S. 2 (2004), p. 137-161 [les vierges sacrifiées le sont moins en raison de leur pureté physique que de leur absolue fidélité à l'oikos paternel; la méthode de genre ici développée permet d'illustrer un aspect problématique de l'étude de la sexualité dans le monde grec].

SHAYA Josephine, « The Greek temple as a museum. The case of the legendary treasure of Athena from Lindos », AJA 109 (2005), p. 423-442 [analyse de la fonction culturelle des trésors de temples hellénistiques fondée sur le cas remarquable de l'inscription dite "Chronique de Lindos $\gg]$.

SimANTONI-BOURnia Eva, «Kyprische Einflüsse I. Ein anthropomorphes Gefäß aus Iria auf Naxos », MDAI(A) 119 (2004), p. 33-54 [découverte d'un lécythe anthropomorphe daté du début du vII s. av. J.-C. consacré dans le sanctuaire d'Hyria; étude des parallèles et mise en relation de l'objet avec une « déesse orientale de la fertilité » du type Astarté]. 
SIMON Erika, « Heilende Heroen », ARG 6 (2004), p. 39-43 [au départ d'une image de Jason soignant les yeux de Phinée par imposition des mains, étude de ce type d'intervention guérisseur chez d'autres héros].

SINEUX Pierre, « Le dieu ordonne. Remarques sur les ordre d'Asklépios dans les inscriptions de Lébèna (Crète) », Kentron 20 (2004), p. 137-146 [les mentions d'ordres dans des textes d'action de grâces ou des catalogues de récits de guérison ne sont pas de simples ornements mais expriment une manifestation de la puissance du dieu qui peut donc s'exercer en dehors du contexte rituel de l'incubation et hors de la sphère de guérison proprement dite].

SINN Ulrich, "Poseidon in Olympia », Nikephoros 17 (2004), p. 45-52 [synthèse des données relatives aux honneurs rendus à Poséidon à Olympie; le maigre butin est étonnant pour un dieu censé être dominant dans le Péloponnèse; rôle des Éléens dans cette occulation].

SIRANO Francesco, «Immagini di divinità da contesto domestico a Cos: la documentazione dagli scavi italiani ", MEFRA 116 (2004), p. 953-981 [examen des sculptures provenant de 3 espaces domestiques du III $^{\text {e }}$ s. ap. J.-C. qui montre que l'usage des statues divines par les élites de l'île renvoie à des attitudes et des goûts culturels traditionnels, mâtinés de philosophie].

Sosin Joshua D., « Unwelcome dedications: Public law and private religion in Hellenistic Laodicea by the sea », CQ 55 (2005), p. 130-139 [remarquable étude des implications de IGLS IV, 1261, un décret qui met en jeu la relation entre les questions économiques (la taxation des dédicaces sur sol civique et non sur sol privé) et les questions religieuses (le scrupule des desservants qui ne veulent pas limiter les dédicaces et se tournent vers l'autorité publique)].

SPEYER Wolfgang, «Die Offenbarungsübermittlung und ihre Formen als mythische und geschichtliche Anschauung », GB 24 (2005), p. 31-43.

SPEYER Wolfgang, «Zur Erfahrung der göttlichen Macht in der Religionsgeschichte des Altertums », Philotheos 5 (2005), p. 139-153.

STAGER Jennifer J.M., " 'Let no one wonder at this image'. A Phoenician funerary stele in Athens », Hesperia 74 (2005), p. 427-449 [analyse approfondie de cette stèle mise au jour au XIX ${ }^{e}$ s. sur le plan du bilinguilisme, du symbolisme religieux et de la définition de soi]

STRASSER Jean-Yves, «Les Antôninia Pythia de Rome », Nikephoros 17 (2004), p. 181-220 [il ne s'agit pas de concours grecs fondés par Élagabal, mais probablement par Caracalla; ils n'ont selon toute vraisemblance été célébrés qu’une seule fois, en 214].

SZIDAT Sabine, «Die „Buckel' der Artemis Ephesia - Zur Bedeutung des Motivs und zu seinen ikonographischen Vorläufern », JDAI 119 (2004), p. 83-129 [étude approfondie des parallèles iconographiques de la "polymastie » de l'Artémis d'Éphèse; il s'agirait d'une évocation de chaînes de montagnes et qualifierait la déesse comme telle, outre ses aspects solaires].

TALloen Peter, WAelkens Marc, «Apollo and the emperors (I). The material evidence for the imperial cult at Sagalassos », AncSoc 34 (2004), p. 171-216.

TAlloen Peter, WAelkens Marc, «Apollo and the emperors (II). The evolution of the imperial cult at Sagalassos », AncSoc 35 (2005), p. 217-249.

Thom Johan C., « Doing justice to Zeus: On texts and commentaries », AClass 48 (2005), p. 1-21 [l'Hymne à Zeus de Cléanthe combine différentes traditions et produit une nouvelle expression de religion philosophique dont il importe de comprendre le caractère agrégatif].

Tiverios Michalis, "Artemis, Dionysos und eleusinische Gottheiten », MDAI(A) 119 (2004), p. 148-162 [Hérodote (II, 156) fait référence à la tradition «égyptienne » qui fait naitre Artémis de Dionysos et de Déméter, et qu'aurait reprise Eschyle; certains vases attiques pourraient également en témoigner, qui représentent Déméter, Dionysos, Artémis et Korè].

TodouA Maïa, « Empédocle : empêche-vents ou dompteur des mauvais génies ? Réflexions autour du fr. 111 Diels-Kranz », BAGB (2005), p. 49-81 [de portée magique, ce passage témoigne de l'aspect religieux de la pensée d'Empédocle; rapprochements avec Éole, mais aussi avec Salmonée, le « faiseur de pluie »; le Papyrus de Strasbourg conforte cette interprétation].

Tremel Jan, « Die Befragung des Orakels durch Athleten », Nikephoros 17 (2004), p. 111-118 [des sites oraculaires étaient aussi le siège de rencontres agonistiques; les oracles pouvaient aider 
les athlètes à prendre une décision : une inscription mise au jour au Sarapieion de Milet montre l'implication de l'oracle de Didymes].

VALDÉS GUÍA Miriam, « Boletín bibliográfico. La constitución de la religión cívica en Atenas arcaica (Parte primera) », Ilu 9 (2004), p. 281-348.

VALDÉS GUÍA Miriam, « La batalla de Sepea y las Hybristika. Culto, mito y ciudadanía en la sociedad argiva », Gerión 25 (2005), p. 101-114.

VALVERDE SÁNCHEZ Mariano, «El mito de Idomeneo, de la épica antigua a la tragedia moderna », Myrtia 20 (2005), p. 265-294.

VAN 'T WOUT P.E., « Amphiaraos as Alkman: Compositional strategy and mythological innovation in Pindar's Pythian 8.39-60 », Mnemosyne 49 (2006), p. 1-18 [le discours du devin prendrait place au moment de son hérö̈sation et non pendant la $2^{\mathrm{e}}$ expédition contre Thèbes].

VAN BREMEN Riet, "Leon son of Chrysaor and the religious identity of Stratonikeia in Caria », YCS 31 (2002), p. 207-244.

VIELLEFON Laurence, « Les mosaïques d'Orphée dans les maisons de l'Antiquité tardive : fonctions décoratives et valeurs religieuses », MEFRA 116 (2004), p. 983-1000 [la popularité d'Orphée en contexte païen et chrétien révèle un idéal plus mystique et spirituel correspondant aux nouvelles aspirations religieuses de la fin de l'Antiquité].

VON MOOCK Derk W., « Delische 'Rundaltäre'. Zur Ausstattung hellenistischer Grabbezirke auf Rheneia ", $M D A I(A) 119$ (2004), p. 373-390 [ces autels ronds à guirlandes portaient des stèles funéraires et s'inscrivaient dans le cadre du culte aux morts et de sa portée mémorielle].

WARMAN L., « Hope in a jar », Mouseion ser. 3, 48 (2004), p. 107-119.

WeILER Ingomar, « Theodosius I. und die Olympischen Spielen », Nikephoros 17 (2004), p. 53-75 [synthèse des données relatives aux mesures prises à l'encontre des concours par les empereurs Théodose I, Théodose II et Justin I].

WEST Martin L., « Archilochus and Telephos », ZPE 156 (2006), p. 11-17.

WEST Martin L., «Odyssey and Argonautica », CQ 55 (2005), p. 39-64 [les poètes des deux œeuvres ont été influencés, indépendamment, par une version grecque de l'épopée de Gilgamesh].

WYLER Stéphanie, «'Dionysos domesticus' : les motifs dionysiaques dans les maisons pompéiennes et romaines (II ${ }^{\mathrm{e}}$ s. av. $-\mathrm{I}^{\mathrm{er}}$ s. ap. J.-C.) », MEFRA 116 (2004), p. 933-951 [étude de l'usage culturel des images dionysiaques en contexte domestique, évoquant un mode de vie à l'hellénistique, centré sur la luxuria et sur l'otium].

WYLER Stéphanie, «Le décor dionysiaque de la villa de la Farnésine : l'art de faire grec à Rome », Mètis N.S. 3 (2005), p. 101-129 [le dionysisme exploité dans la villa est un paradigme culturel savamment exploité]. 